\title{
The occurrence of Cryptosporidium parvum in dairy calves and the influence of management practices
}

\begin{abstract}
Cryptosporidium species infections are associated with the clinical manifestation of diarrhea, which can possibly generate economic losses associated with morbidity and mortality depending on the type of property management used. Additionally, animals with diarrhea eliminate large quantities of oocysts via the feces, thus contributing to the dispersion of oocysts in the environment and favoring the infection of susceptible hosts. The study aimed to determine the rate of Cryptosporidium parvum infection in 79 calves under one year of age at a dairy farm in southern Rio de Janeiro state, Brazil, in association with the cattle raising management practices. During the study period, two different management practices (A and B) were adopted. Under practice A, $21.9 \%$ of the samples (7/32) were Cryptosporidium spp. positive according to microscopic diagnosis. After switching to practice $\mathrm{B}$, the percentage of infected animals decreased to $8.5 \%(4 / 47)$. Although diarrhea was reported in $13.9 \%(11 / 79)$ of the calves studied, there was no correlation with Cryptosporidium spp. infection $(\mathrm{p}>0.05)$. Cryptosporidium parvum, which is considered to have high zoonotic potential, was identified in this study. This fact is of great importance with regard to public health because of the environmental contamination of the area surrounding the property, which increases the risk to both human and animal populations. Additionally, the implementation of some changes in farm management practices contributed to a decrease in the percentage of infected animals, thereby reducing the dissemination of oocysts in the environment.
\end{abstract}

Keywords: calves, cryptosporidium parvum, management, diarrhea, environmental contamination
Volume 2 Issue 2 - 2015

\author{
Melissa Carvalho Machado do Couto,' \\ Marcelo de Freitas Lima, ${ }^{2}$ Marcus Sandes \\ Pires, 'Teresa Cristina Bergamo do Bomfim' \\ 'Department of Animal Parasitology, Federal Rural University of \\ Rio de Janeiro, Brazil \\ ${ }^{2}$ Department of Chemistry, Federal Rural University of Rio de \\ Janeiro, Brazil
}

\begin{abstract}
Correspondence: Teresa Cristina Bergamo do Bomfim, Federal Rural University of Rio de Janeiro, Department of Animal Parasitology, Veterinary Institute, BR 465, Km 07, 23.890000, Seropédica-Rio de Janeiro, Brazil, Tel +55 2l 2682 1617, Fax +552126821617 Email tcbb@ufrrj.br, tcbb@superig.com.br
\end{abstract}

Received: January 21, 2015 | Published: May 06, 2015
Abbreviations: PCR, polymerase chain reaction; OR, odds ratio: $\mathrm{CI}$, confidence interval

\section{Introduction}

Dairy cattle production is considered one of the main agricultural activities worldwide. A major problem associated with milk production is related to the sanitary aspects of cattle rearing. Parasitic diseases are particularly highlighted because these have been reported to be the most prevalent. ${ }^{1}$ Among the diseases caused by protozoa, cryptosporidiosis has been emphasized given its responsibility for considerable economic losses in the production chain. These losses are related to the disease pathology that can cause severe diarrhea and intestinal malabsorption syndrome in an age, host immune status- and Cryptosporidium species-dependent manner and can lead to morbidity and even mortality in calves. ${ }^{2-6}$

Diarrheal outbreaks among cattle result in financial losses because the symptoms are directly related to reduced weight gain, decreased productivity and increased mortality. ${ }^{7-9}$ Therefore, bovine cryptosporidiosis is widely studied, given the economic impact of diarrhea on the production chain. ${ }^{10}$ Out of 26 valid species of the genus Cryptosporidium, C. parvum is responsible for causing clinical symptoms, especially in calves approximately one month of age..$^{7,8,11,12}$

The duration of diarrhea in Cryptosporidium-infected calves depends on several factors, including the level of environmental contamination, virulence, infectivity of the involved species, host susceptibility and age at first infection. ${ }^{13}$

The prevalence of $C$. parvum infection varies widely in cattle, with herd positivity rates ranging from $13-100 \%$, especially before one month of age. ${ }^{11,14-17}$ This variation in prevalence might be directly influenced by certain factors such as the host age, study area characteristics and study methodology. ${ }^{11,13,15,18}$

In Brazil, the prevalence of Cryptosporidium spp. infection in dairy cattle ranges from $0.6-72.13 \%$ and C. parvum is most prevalent in animals less than two months of age. ${ }^{19-21}$

Property sanitation can directly influence the route of cryptosporidiosis transmission. Poor sanitary conditions in the environments where animals are raised, particularly in rearing pens, can increase the animals' infection risk. ${ }^{22}$ Therefore, the use of appropriate management practices is required to prevent cryptosporidiosis outbreaks. ${ }^{7}$ The use of simple management practices such as raising calves in individual pens have led to reduced infection rates and consequently reduced environmental contamination. ${ }^{22}$

Given the lack of studies regarding the influence of dairy cattle management on Cryptosporidium spp. infection and the relevance of the results previously obtained ${ }^{21}$ in a study conducted in the state of Rio de Janeiro, Brazil, some previously published data were used as the basis for the present study, which aimed to determine the role of a few practical management changes in the occurrence of $C$. Parvum in calves up to 1-year-old raised in a dairy farm in the state of Rio de Janeiro, Brazil.

\section{Materials and methods}

The C. Parvum infection occurrence was studied in 79 Holstein crossbred calves up to one year old for a 15 month period. All studied 
calves were born and raised in the dairy farm at which the study was conducted, that was small in size and located in the Vale do Paraíba microregion (22 $42^{\prime} 30.17^{\prime \prime} \mathrm{S}$ and $\left.43^{\circ} 46^{\prime} 51.80^{\prime \prime} \mathrm{W}\right)$, Rio de Janeiro state, Brazil.

The study quasi-experimental design was conducted in this dairy farming property. All 79 Holstein crossbred calves were separated into groups according to their feeding behavior as pre-weaned and post-weaned calves. The pre-weaned group consisted in calves up to 2 months old that were feed with milk, bovine ration and hay. The post-weaned group consisted in bovines older than 2 months old up to one year old that were similarly fed as the first group described, except for the milk ingestion.

Two different management practices (A and B) were used during sample collection; which primarily consisted in a different way of housing the calves and the modification in the height of the feeders. In both groups, there were animal with pre-weaned and post-weaned calves.

At the first stool sample collection, the animals were housed in collective rearing pens in a semi-open facility which the sanitary conditions were poor and inadequately managed (Management A), with the drinkers and feeders near the floor. These stalls were generally cleaned once in a day, being constantly humid, due the absence of sunlight incidence. In regard of the cleaning management, sometimes could be seen feces spread all over the floor. The collective pens were located near to a river that crossed the dairy farm. When the rearing pens were washed, the runoff water was drained into the river.

Later, changes to the facilities and calf management practices (Management B) were suggested to the owner. The calves were moved from collective rearing pens of a semi-open facility to individual hutches located in a wider area without humidity. Besides the changes in the facility area, the drinkers and the feeders were installed in a higher place in the rearing pen. However, despite the new facility have changed place, the cleaning management stills the same and the runoff water continued to be drained into the river. This new facility was located near the area where the adult animals were housed.

In both cases, the fecal material was collected directly from the rectal ampulla of the animals, labeled and stored at $4^{\circ} \mathrm{C}$ until processing in the Laboratory of Protozoology, Department of Animal Parasitology, Federal Rural University of Rio de Janeiro (Universidade Federal Rural do Rio de Janeiro; UFRRJ).

Most of the collected fecal samples were within the normal range; however, some calves exhibited clinical manifestations of diarrhea In addition, the occurrence of diarrhea in calves was evaluated and compared it with the positivity occurrence of Cryptosporidium sp. in animals.

In the laboratory, the samples were cataloged, homogenized, filtered, processed according to the sedimentation and flotation technique ${ }^{23}$ and analyzed for the presence of Cryptosporidium spp. oocysts using light microscopy with and without phase-contrast.

All positive samples were processed using molecular techniques to confirm the presence of Cryptosporidium spp. infection; for detection and differentiation of the involved Cryptosporidium species and genotypes. The genomic DNA was extracted using the QIAamp DNA Stool Mini Kit (Qiagen ${ }^{\circledR}$, Venlo, The Netherlands) according to the manufacturer's recommendations, with slight modifications. ${ }^{24}$
Polymerase Chain Reaction (PCR) was performed immediately after DNA extraction. Two target genes, $18 S$ and GP60, were used to amplify of the Cryptosporidium spp. genomic DNA. Diagnosis of the infectious species and subtypes collected from the animals was performed via PCR according to the method described in a previous study, ${ }^{24}$ which used nested PCR reactions with known primers for the $18 S$ gene $^{25}$ and for the GP60 gene. ${ }^{26}$

All of the primary PCR and nested-PCR products generated during the amplification of both target genes were visualized on a $1 \%$ agarose gel stained with Gelred ${ }^{\mathrm{TM}} .{ }^{24}$ The protocols used for sample purification, genotypic characterization and cloning were conducted according to the method previously described by the authors. ${ }^{21}$

To evaluate the effect of the type of livestock management front positivity for Cryptosporidium sp. in dairy calves, a simple logistic regression model was performed to correlate the dependent variable (diagnosis for Cryptosporidium sp.) in dichotomous qualitative levels (negative $=0$ and positive $=1$ ) with the variable independent (zootechnical management $\mathrm{A}=0$ and $\mathrm{B}=1$ ), also the dichotomous level for each category evaluated, at a $5 \%$ level of significance. Final logistic regression model was analyzed by the likelihood ratio. As null hypothesis, the livestock management does not influence the Cryptosporidium sp. positivity rate in calves. This analysis was performed using the R statistical software package. ${ }^{27}$

The Phi (Ø) correlation coefficient was used to assess the relationship between the positivity of animals by Cryptosporidium sp. (negative $=0$ and positive $=1$ ) and occurrence of diarrhea in calves (yes $=1$ and no=0), also with the $5 \%$ significance level. In this analysis, the null hypothesis is that there is no correlation between the occurrence of diarrhea and infection by Cryptosporidium sp. in dairy calves. It was used for this analysis the statistical program Bio Estat 5.0.28

\section{Results}

A total of 79 Holstein crossbred calves were evaluated in the present study. During the first fecal material collection period (Management A), 32 calves were evaluated. After performing the suggested modifications, a second fecal material collection (Management B) was performed to evaluate another 47 animals.

The 79 calves from the dairy farm where the study was conducted were evaluated for the presence and absence of diarrhea and accessed via microscopic and molecular techniques for Cryptosporidium spp. infection. A clinical evaluation revealed the presence of diarrhea in $13.9 \%(11 / 79)$ of the calves; however, microscopy revealed that only $2.5 \%(2 / 79)$ of these animals were infected with Cryptosporidium spp. (Table 1). In addition to Cryptosporidium spp., other diarrheainducing gastrointestinal protozoa were observed in the fecal parasite tests, including Eimeria spp. and Giardia intestinalis. Eimeria spp. was more frequently present in both animals with feces of normal consistency and those with diarrhea (Table 2). Using microscopy with and without phase-contrast, Cryptosporidium spp. infections were diagnosed in $13.9 \%(11 / 79)$ of the studied calves studied; of these, $21.9 \%(7 / 32)$ belonged to the group under Management A, and $8.5 \%(4 / 47)$ belonged to the group under Management B (Table 1).

Subsequently, all microscopically determined Cryptosporidium spp. positive samples were subjected to diagnostic confirmation via nested-PCR for $18 S$ gene amplification. Among the studied calves, $81.8 \%(9 / 11)$ of the positive microscopic diagnosis were confirmed by 
nested-PCR; $45.5 \%(5 / 11)$ were from the group under Management A, and $36.4 \%(4 / 11)$ were from the group under Management B (Table $1)$.
All nine $18 S$ gene PCR-positive samples were sequenced to yield good quality products, thus allowing the identification of three distinct Cryptosporidium species, one of which was C. Parvum, a species with high zoonotic potential (Table 1).

Table I Cryptosporidium spp. positive fecal samples from calves less than 12 months of age, the presence or absence of diarrhea and the results of microscopic and molecular diagnostic analyses

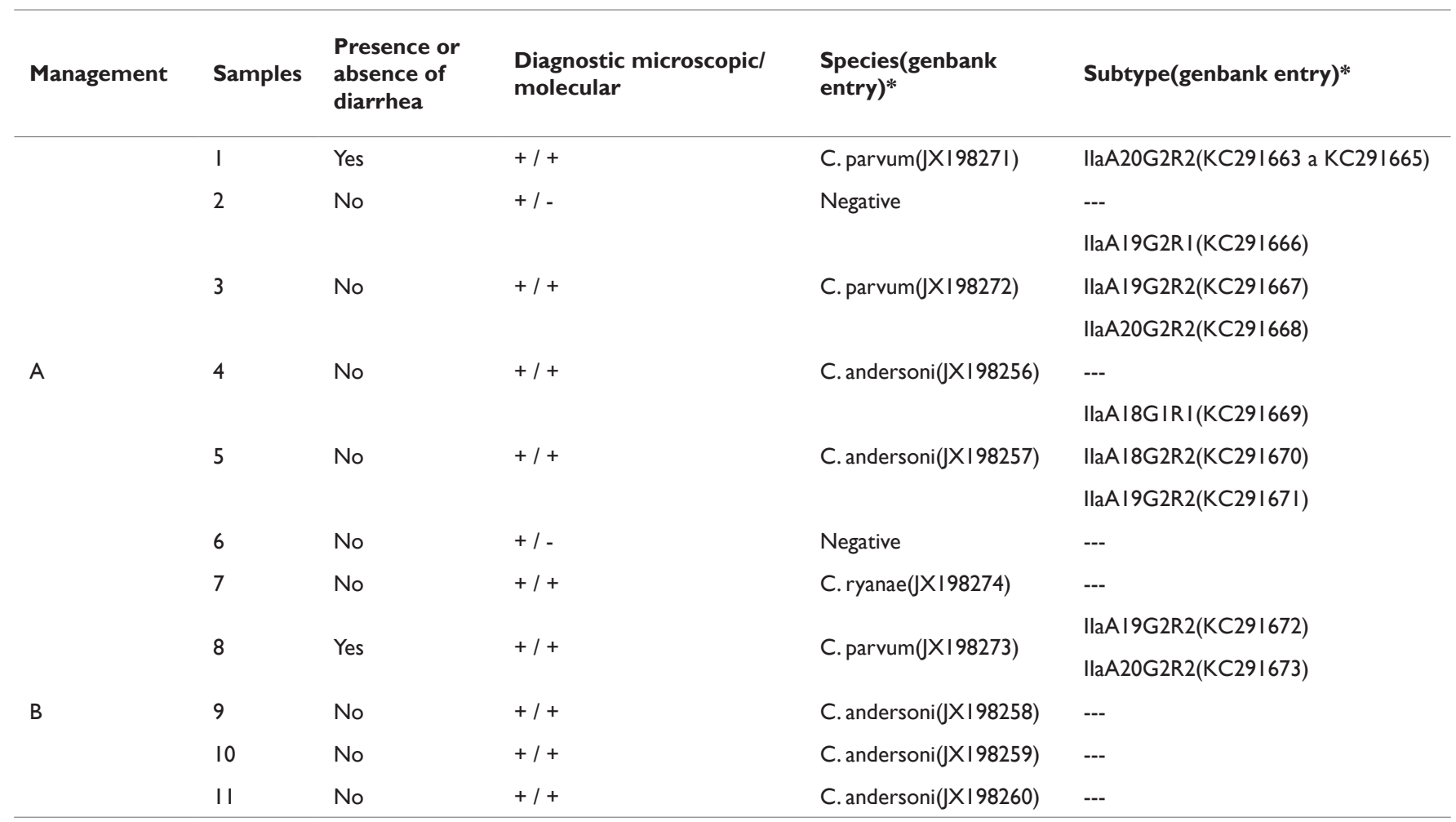

*Data adapted from Couto et al. ${ }^{21}$

Table 2 Microscopic diagnosis of gastrointestinal protozoa using the technique of centrifugation and flotation in a saturated sugar solution for fecal samples of calves with or without diarrhea from a dairy farm in Vale do Paraiba, Rio de Janeiro, Brazil

\begin{tabular}{llll}
\hline Diarrheal feces(N = I I) & \multicolumn{3}{l}{ Normal feces(N = 68) } \\
\hline Negative & $3(27.3 \%)$ & Negative & $\mathrm{I}(25.0 \%)$ \\
Cryptosporidium spp. & $2(18.2 \%)$ & Cryptosporidium spp. & $5(7.4 \%)$ \\
Eimeria spp. & $5(45.4 \%)$ & Eimeria spp. & $40(58.8 \%)$ \\
Giardia intestinalis & $\mathrm{I}(9.1 \%)$ & Giardia intestinalis & $\mathrm{I}(\mathrm{I} .4 \%)$ \\
Mixed Infection: & Negative & Mixed Infection: & $5(7.4 \%)$ \\
Cryptosporidium spp.+Eimeriaspp. & Cryptosporidium spp.+ Eimeriaspp. \\
\hline
\end{tabular}

After sequencing the samples according to the $18 S$ target gene, all nine Cryptosporidium sp.-positive samples were subjected to GP60 gene amplification for the identification of C. Parvum and related subtypes. Of these samples, 44.4\%(4/9) exhibited gene amplification, indicating the presence of C. Parvum (Table 1).

After sequencing, however, overlapping peaks were observed in the chromatograms; these were most likely due to the presence of multiple sequences amplified within the same sample, thus preventing the reliable identification of C. Parvum subtypes. Therefore, DNA fragment individualization was performed via cloning. Subsequently, several colonies were obtained from each sample, of which three were randomly selected for further sequencing with universal primers. A total of 12 sequences were obtained, and of these, all but one sample contained the C. Parvum subtype involved in the calves' infections (Table 1).

In this study, $21.9 \%(7 / 32)$ of the calves raised under Management A were infected with at least one Cryptosporidium spp. After changing to Management B, this percentage decreased, and the infection rate was only $8.5 \%$ (4/47). After analyze of simple logistic regression, no significant difference $(\mathrm{p}=0.10)$ was detected between the two 
management types used at the farm with regard to Cryptosporidium spp. infection. Similarly, the OR indicated that although animals raised under Management A were threefold more likely to harbor a Cryptosporidium spp. infection, the confidence interval (CI) showed no relationship between these study variable (CI: $0.80-11.30$ ) Table 3 .
Similarly, no statistical correlation was observed between Cryptosporidium spp. positivity and the presence of diarrhea in the calves $(\mathrm{p}=0.97 ;$ Phi coefficient=0.04). The percentage of Cryptosporidium spp.-positive animals with diarrhea was $18.2 \%(2 / 11)$, which was slightly higher than the percentage in calves without diarrhea $(13.2 \%$; 9/68).

Table 3 Logistic regression model of the frequency of positive on dairy calves Cryptosporidium spp. depending zootechnical management practices in property, in the state of Rio de Janeiro, Brazil

\begin{tabular}{|c|c|c|c|c|c|c|c|c|}
\hline \multirow{2}{*}{ Variables } & \multirow{2}{*}{$\mathbf{N}$} & \multicolumn{2}{|c|}{ Positive } & \multicolumn{5}{|c|}{ Logistic regression model } \\
\hline & & (n) & $(\%)$ & $C(B)$ & SP & $\mathbf{P}$ & OR & $\mathrm{Cl} 95 \%$ \\
\hline Model constant & & & & $-2,374$ & 0,522 & 0,000 & & \\
\hline \multicolumn{9}{|c|}{ Management practices in property } \\
\hline Type A & 32 & 7 & 21.87 & 1.101 & 0.675 & 0.103 & 3.01 & $(0,80-|I, 3|)$ \\
\hline Type B* & 47 & 4 & 8.51 & - & - & - & - & - \\
\hline TOTAL & 79 & II & 13.92 & - & - & - & - & - \\
\hline
\end{tabular}

$\mathrm{N}$, total number of animals; (n), number of positives animals; (\%), positive rates; C (ß), coefficient category; SP, standard error; $\mathrm{P}$, $\mathrm{P}$-value; OR, Odds ratio; CI, confidence interval. *Reference category

\section{Discussion}

The difference between the results obtained in the present study, using microscopic and molecular diagnostic techniques remains a controversial subject within the scientific community. Some researchers have reported the high sensitivity of PCR-mediated oocyst detection, ${ }^{15,29}$ whereas others have reported better results when using microscopy. ${ }^{9}$ Several hipothesis have been considered to explain the results of the present study. Among the major hipothesis, we can cite the low parasite loads present in some samples, the non-homogeneous oocyst distribution in the fecal material and the presence of inhibitors that might have led to underestimations of the PCR results. Regarding the low parasite loads, some authors ${ }^{9}$ mentioned that a low oocyst recovery from fecal material could yield a low percentage of positive results when using molecular techniques.

In addition to the issue of low numbers of oocysts and the PCRmediated detection of Cryptosporidium spp., is supposed that the heterogeneous oocyst distribution in the feces might occasionally yield false-negative results. ${ }^{30}$ These authors also suggested that a large numbers of oocysts observed via microscopy would not guarantee diagnostic confirmation by PCR because the oocysts would have been damaged before and during the extraction process, possibly leading to reduced numbers of the available DNA targets required for PCR and thereby reducing its effectiveness. Another explanation might be the presence of high concentrations of inhibitory substances in the calves' fecal material, which would limit the molecular diagnostic efficiency. ${ }^{31}$ Although the present study did not focus on a comparison of the diagnostic techniques, the previous authors suggested that one technique would complement the other.

The $18 S$ and GP60 gene-based molecular diagnoses facilitated the identification of different $C$. Parvum subtypes belonging to the IIa subtype family. In Brazil, studies on human and animal infections with different $C$. Parvum subtypes are scarce. In a study performed in the state of São Paulo, Brazil, ${ }^{32}$ the presence of a zoonotic C. Parvum subtype (IIaA15G2R1) was diagnosed in capybaras. In another study conducted in several municipalities in the state of São Paulo, ${ }^{33}$ the zoonotic subtype IIaA15G2R1 was diagnosed in cattle, unlike the results obtained in the present study.
To date, there have been no reports in South America of human infections resulting from members of the subtype IIa family, which are commonly found in cattle. However, a study in Peru reported that children were infected only by the C. Parvum subtype IIc family, diagnosing the subtypes IIcA5G3a, IIcA5G3b and IIcA5G3c. ${ }^{34}$

On dairy farms, disease associated with C. Parvum infection occurs primarily in young calves, ${ }^{35}$ being reportedly as one of the major species that causes diarrhea, a clinical symptom characteristic of cryptosporidiosis. ${ }^{5,36,37}$ In the present study, C. Parvum was the only diagnosed infectious Cryptosporidium spp. in calves with diarrhea, a finding that agreed with those of the above-cited authors. However, diarrhea was also observed in Cryptosporidium spp.-negative animals, suggesting that this symptom might be consequent to infections by other gastrointestinal etiologic agents, including Eimeria spp. and Giardia intestinalis. ${ }^{8,38}$ Regarding the present study, we also suggest the possibility of false-negative parasitological tests, especially in animals with diarrheal feces because these animals might have eliminated the infective form of the protozoa at a low parasitic load or intermittently, a finding that has also been reported in other research. ${ }^{8}$

Concomitant Cryptosporidium spp. and Eimeria spp. infections were only observed in fecal samples of normal consistency, in contrast with the findings of other researchers, who observed a higher co-infection incidence in diarrheic fecal samples. The authors of the current study agree with the hypothesis suggested in a previous research, ${ }^{8}$ in which the calves likely experienced diarrhea during a period prior to sample collection, after which oocyst shedding of both parasites continued through the collection date regardless of the fecal consistency.

The importance of reducing the exposure of calves to Cryptosporidium genus protozoa, particularly C. Parvum, has been described before, ${ }^{39}$ who highlighted the cattle rearing area sanitation level and infected animal management and care as relevant factors associated with infection. The significance of adequate management practices in dairy farms was also described as potentially important predictors of the prevalence of shedding C. Parvum oocysts in dairy calves under 1 month of age. ${ }^{35}$ 
In this study, management practices that were adopted at the farm, particularly the calves' housing location, were evaluated, and a reduction in the percentage of infected animals was observed after some of the management practices were changed along with a change in the rearing pen from location A to location B.

The Cryptosporidium spp. infection percentage (21.9\%) observed in the calves is related to the poor sanitation encountered during the use of Management A. These poor conditions included the permanent presence of feces in the pens, which might have favored infection dissemination along with the high environmental humidity. Although not shown the statistical interaction between the type of animal management and Cryptosporidium sp. positivity $(P \geq 0.05)$, the number of infected animals was twofold higher under Management A. However, for the data analyzed, if the strength of association is reduced for $90 \%$ of significance, was observed that animals raised under the condition of Management A were the most affected by this infection. Therefore, it is possible that with a larger sample number, the influence of the management type on the positivity can be better highlighted. It is therefore possible that after transferring the animals to another location (Management B), the observed reduction in the infection incidence $(8.5 \%)$ might have been related to the reduced environmental humidity and the use of individual pens. These changes might have facilitated a reduction in the percentage of infected calves by minimizing contact between the animals and, consequently, the possible transmission between the animals.

Changes in the placement of the feeders and drinkers within the new facility (Management B) might also have helped to reduce the percentage of infected animals. The feeders and drinkers were placed relatively higher than their locations under Management A, a practice that most likely reduced the likelihood of fecal water and food contamination and, consequently, infection.

The absence of correlation between the occurrence of diarrhea and positivity for Cryptosporidium sp. can be related to other factors inherent to the animals such as the presence of other gastrointestinal parasites and/or concomitant infections.

The incidence of Cryptosporidium spp. infection in dairy cattle was also studied before. ${ }^{40}$ The authors of that study evaluated several factors associated with infections in calves, where one of the most relevant was the property management and the use of shared facilities.

During the use of Management A, the feces from calves housed in collective pens were directly discarded into the river that passed through the property. This management practice can lead to several problems, including environmental contamination and the occurrence of possible cryptosporidiosis outbreaks in other areas. A similar situation was observed in Malaysia, ${ }^{2}$ where the authors described the contamination of rivers near farms due to inadequate cattle manure management practices.

Depending on the animals' parasite loads and the species/subtypes, disease outbreaks can even occur in humans. This outcome was suggested by the presence of the C. Parvum subtype (IIa) family, which has high zoonotic potential, in the studies farm.

Despite changes in the management practices on the farm, the environmental contamination issue was not resolved. This is because under Management B, the feces from the individual pens were also carried by rainwater to the river that passed through the property, thus maintaining Cryptosporidium oocyst dissemination and continuing to facilitate the potential problem described above. The importance of environmental contamination from the organic waste produced on farms has been reported before in other studies, ${ }^{41,42}$ and these earlier findings agreed with those described in this study. Although there was a reduction in the percentage of Cryptosporidium spp.-infected calves, further studies are required to demonstrate whether simple changes in the management practices of a particular property could help to reduce the incidence of cryptosporidiosis in dairy cattle.

\section{Acknowledgements}

We thank the National Council for Scientific and Technological Development (ConselhoNacional de DesenvolvimentoCientífico e Tecnológico - CNPq) and 'Carlos ChagasFilho' Foundation for Research Support of the State of Rio de Janeiro (Fundação Carlos Chagas Filho de Amparo à Pesquisa do Estado do Rio de Janeiro FAPERJ) for the financial support that made this study possible.

\section{Conflict of interest}

Author declares that there is no conflict of interest.

\section{References}

1. Lucena RB, Pierezan F, Kommers GD, et al. Doenças de bovinos no Sul do Brasil: 6.706 casos. Pesq Vet Bras. 2010;30(5):428-434.

2. Farizawati S, Lim YAL, Ahmad RA, et al. Contribution of cattle farms towards river contamination with Giardia cysts and Cryptosporidium oocysts in Sungai Langat Basin. Trop Biomed. 2005;22(2):89-98.

3. Broglia A, Reckinger S, Cacció SM, et al. Distribution of Cryptosporidium parvum subtypes in calves in Germany. Vet Parasitol. 2008;154(1-2):8-13

4. Ollivet TL, Nydam DV, Bowman DD, et al. Effect of nitazoxanide on cryptosporidiosis in experimentally infected neonatal dairy calves. $J$ Dairy Sci. 2009;92(4):1643-1648.

5. Chako CZ, Tyler JW, Schultz LG, et al. Cryptosporidiosis in people: It's not just about the cows. $J$ Vet Intern Med. 2010;24(1):37-43.

6. Xiao L. Molecular epidemiology of cryptosporidiosis: An update. Exp Parasitol. 2010;124(1):80-89.

7. Nydam DV, Mohammed HO. Quantitative risk assessment of Cryptosporidium species infection in dairy calves. J Dairy Sci. 2005;88(11):3932-3943.

8. Gulliksen SM, Jor E, Lie KI, et al. Enteropathogens and risk factors for diarrhea in Norwegian dairy calves. J Dairy Sci. 2009;92(10):5057-5066.

9. Díaz P, Quílez J, Chalmers RM, et al. Genotype and subtype analysis of Cryptosporidium isolates from calves and lambs in Galicia (NW Spain). Parasitology. 2010;137(8):1187-1193.

10. Imre K, Dărăbus G. Distribution of Cryptosporidium species, genotypes and $C$. parvum subtypes in cattle in european countries. Sci Parasitol. 2011;12(1):1-9.

11. Starkey SR, Zeigler PE, Wade SE, et al. Factors associated with shedding of Cryptosporidium parvum versus Cryptosporidium bovis among dairy cattle in New York. J Am Vet Med Assoc. 2006;229(10):1623-1626.

12. Cardoso JMS, Silveira FL, Araújo AJUS, et al. Ocorrência de Cryptosporidium spp. em um rebanho bovino leiteiro no município de Caçapava, Estado de São Paulo, Brasil. Rev Bras Parasitol Vet. 2008;17(Suppl 1):239-242.

13. Feitosa FLF, Shimamura GM, Roberto T, et al. Importância de Cryptosporidium spp. como causa de diarreia em bezerros. Pesq Vet Bras. 2008;28(10):452-456. 
14. Hamnes IS, Gjerde B, Robertson L. Prevalence of Giardia and Cryptosporidium in dairy calves in three areas of Norway. Vet Parasitol. 2006;140(3-4): 204-216.

15. Santín M, Trout JM, Fayer R. A longitudinal study of cryptosporidiosis in dairy cattle from birth to 2 years of age. Vet Parasitol. 2008;155(1-2):15-23.

16. Silverlås C, Emanuelson U, de Verdier K, et al. Prevalence and associated management factors of Cryptosporidium shedding in 50 Swedish dairy herds. Prev Vet Med. 2009;90(3-4):242-253.

17. Rieux A, Chartier C, Pors I, et al. Dynamics of excretion and molecular characterization of Cryptosporidium isolates in pre-weaned French beef calves. Vet Parasitol. 2014;195(1-2):169-172.

18. Brook E, Hart CA, French N, et al. Prevalence and risk factors for Cryptosporidium spp. infection in young calves. Vet Parasitol. 2008;152(1-2):46-52.

19. Meireles MV. Cryptosporidium infection in Brazil: implications for veterinary medicine and public health. Rev Bras Parasitol Vet. 2010;19(4):197-204.

20. Paz e Silva FM, Lopes RS, Araújo-Júnior JP. Identification of Cryptosporidium species and genotypes in dairy cattle in Brazil. Rev Bras Parasitol Vet. 2013;22(1):22-28.

21. Couto MC, Lima MD, Bomfim TC. New Cryptosporidium parvum subtypes of IIa subfamily in dairy calves from Brazil. Acta Trop. 2014;130(C):117-122.

22. Feitosa FLF, Shimamura GM, Roberto T, et al. Prevalência de criptosporidiose em bezerros na região de Araçatuba, estado de São Paulo, Brasil. Ciênc Rural. 2004;34(1):189-193.

23. Huber F, da Silva S, Bomfim TC, et al. Genotypic characterization and phylogenetic analysis of Cryptosporidium sp. from domestic animals in Brazil. Vet Parasitol. 2007;150(1-2):65-74.

24. Couto MCM, Sudre AP, Lima MF, et al. Comparison of techniques for DNA extraction and agarose gel staining of DNA fragments using samples of Cryptosporidium. Veterinari Medicina. 2013;58(10):535-542.

25. Fayer R, Santín M, Dargatz D. Species of Cryptosporidium detected in weaned cattle on cow-calf operations in the United States. Vet Parasitol. 2010;170(3-4):187-192

26. Sulaiman IM, Hira PR, Zhou L, et al. Unique endemicity of cryptosporidiosis in children in Kuwait. J Clin Microbiol. 2005;43(6):2805-2809.

27. R DEVELOPMENT CORE TEAM. R: A language and environment for statistical computing. Austria: R Fundation for Statistical Computing. 2009.

28. Ayres M, Ayres JR, Ayres DL, et al. BioEstat 5.0-Aplicações Estatísticas nas Áreas das Ciências Biológicas e Médicas. 5th ed. Belém: IOEPA; 2007. p. 364.
29. Bairami Kuzehkanan A, Rezaeian M, Zeraati H, et al. A sensitive and specific PCR based method for identification of Cryptosporidium sp. using new primers from $18 S$ ribosomal RNA. Iran J Parasitol. 2011;6(4):1-7.

30. Magi B, Canocchi V, Tordini G, et al. Cryptosporidium infection: diagnostic techniques. Parasitol Res. 2006;98(2):150-152.

31. Silva DG, Silva DR, Silva PRL, et al. Avaliação da reação em cadeia da polimerase e do isolamento bacteriológico convencional na detecção de Salmonella Dublin em amostras de fezes de bezerros infectados experimentalmente. Arq Bras Med Vet Zootec. 2010;62(3):752-756.

32. Meireles MV, Soares RM, Bonello F, et al. Natural infection with zoonotic subtype of Cryptosporidium parvum in capybara (Hydrochoerushydrochaeris) from Brazil. Vet Parasitol. 2007;147(1-2):166-170.

33. Meireles MV, de Oliveira FP, Teixeira WF, et al. Molecular characterization of Cryptosporidium spp. indairy calves from the state of São Paulo, Brazil. Parasitol Res. 2011;109(3):949-951.

34. Cama VA, Bern C, Roberts J, et al. Cryptosporidium species and subtypes and clinical manifestations in children, Peru. Emerg Infect Dis. 2008;14(10):1567-1574

35. Trotz Williams LA, Martin SW, Leslie KE, et al. Association between management practices and within-herd prevalence of Cryptosporidium parvum shedding on dairy farms in southern Ontario. Prev Vet Med. 2008;83(1):11-23.

36. Santín M Trout JM. Livestock. In: Fayer R, Xiao L. editors. Cryptosporidium and cryptosporidiosis. 2nd ed. Florida, USA: CRC Press; 2007. p. 451-484.

37. Santín M. Clinical and subclinical infections with Cryptosporidium in animals. $N Z$ Vet $J .2013 ; 61(1): 1-10$.

38. Silverlås C, Verdier K, Emanuelson U, et al. Cryptosporidium infection in herds with and without calf diarrheal problems. Parasitol Res. 2010;107(6):1435-1444.

39. Harp JA, Goff JP. Strategies for the control of Cryptosporidium parvum infection in calves. J Dairy Sci. 1998;81(1):289-294.

40. Silva-Junior FA, Carvalho AHO, Rocha CMBM, et al. Fatores de risco associados à infecção por Cryptosporidium spp. eGiardiaduodenalis em bovinos leiteiros na fase de cria e recria na mesorregião de Campos das Vertentes de Minas Gerais. Pesq Vet Bras. 2011;31(8):690-696.

41. Smith HV, Rose B. Waterborne cryptosporidiosis: Current status. Parasitol Today. 1998;14(1):14-22.

42. Barwick RS, Mohammed HO, White ME, et al. Factors associated with the likelihood of Giardia spp. and Cryptosporidium spp. in soil from dairy farms. J Dairy Sci. 2003;86(3):784-791. 